\title{
Research on the Problem of Maximum-Minimum Related to Probability of n Events
}

\author{
Cui Meng and Peiqun $\mathrm{Wu}^{*}$ \\ Beijing Electronic Science and Technology Institute, Beijing 100070, China \\ *Corresponding author
}

\begin{abstract}
The problem of maximum and minimum on the difference between the product of two events' probability and the probability of product of two events has been discussed, in this paper, two events are generalized to $n$ events and the problem is solved by using the method of induction and construction.
\end{abstract}

Keywords-probability; maximum; minimum; inductive method; constructive method

\section{INTRODUCTION}

The maximum- minimum problem of the probability of two events is discussed in [1-3], Proposition 1 discussed the maximum and Proposition 2 discussed the minimum problem.

Proposition 1.

$$
P(A B)-P(A) P(B) \leq \frac{1}{4}
$$

Proposition 2.

$$
-\frac{1}{4} \leq P(A B)-P(A) P(B)
$$

In the paper, we generalized Proposition 1 and Proposition 2 from two events to three events and n events, used inductive method and constructive method to solve the problem, the maximum value of three events is discussed in Proposition 3 ,the maximum value of $\mathrm{n}$ events is discussed in Proposition 4 , the minimum value of three events is discussed in Proposition 5.the minimum value of $\mathrm{n}$ events is discussed in Proposition 6.

\section{THE MAXIMUM VALUE}

Proposition 3.

$$
P(A B C)-P(A) P(B) P(C) \leq \frac{2}{9} \sqrt{3}
$$

Proof.

Assume that

$$
P(A) \geq P(B) \geq P(C)
$$

$$
P(A B C)-P(A) P(B) P(C) \leq P(C)\left(1-(P(C))^{2}\right)
$$

Define $\mathrm{f}(\mathrm{x})$ as follows:

$$
f(x)=x-x^{3}, x \in[0,1], f(x) \leq \frac{2}{9} \sqrt{3}
$$

$$
P(A B C)-P(A) P(B) P(C) \leq \frac{2}{9} \sqrt{3}
$$

$$
\text { if } P(A)=P(B)=P(C)=\frac{\sqrt{3}}{3}, A=B=C \text {, }
$$

the equality is true.

The proof is complete.

Proposition 4.

$$
P\left(\coprod_{i=1}^{n} A_{i}\right)-\prod_{i=1}^{n} P\left(A_{i}\right) \leq\left(\frac{1}{n}\right)^{\frac{1}{n-1}}\left(1-\frac{1}{n}\right)
$$

Proof.

Assume that

$$
\begin{gathered}
P\left(A_{1}\right) \geq P\left(A_{2}\right) \geq \ldots \geq P\left(A_{n}\right), \\
P\left(\prod_{i=1}^{n} A_{i}\right)-\prod_{i=1}^{n} P\left(A_{i}\right) \leq P\left(A_{n}\right)-P^{n}\left(A_{n}\right)
\end{gathered}
$$

Define $\mathrm{f}(\mathrm{x})$ as follows:

$$
f(x)=x-x^{n}, x \in[0,1] .
$$

the maximum value of $f(x)$ is

$$
\left(\frac{1}{n}\right)^{\frac{1}{n-1}}\left(1-\frac{1}{n}\right)
$$

$$
P\left(\prod_{i=1}^{n} A_{i}\right)-\prod_{i=1}^{n} P\left(A_{i}\right) \leq\left(\frac{1}{n}\right)^{\frac{1}{n-1}}\left(1-\frac{1}{n}\right)
$$




$$
\text { if } \begin{gathered}
P\left(A_{1}\right)=P\left(A_{2}\right)=\ldots=P\left(A_{n}\right)=\left(\frac{1}{n}\right)^{\frac{1}{n-1}}, \\
A_{1}=A_{2}=\ldots=A_{n},
\end{gathered}
$$

the equality is true.

The proof is complete.

III. MINIMUM VALUE

Proposition 5.

$$
-\frac{8}{27} \leq P(A B C)-P(A) P(B) P(C)
$$

Proof.

$$
\begin{gathered}
P(A) P(B) P(C)-P(A B C) \\
=P(A) P(B)[P(A B C)+P(\overline{A B} C)]-P(A B C) \\
=P(A) P(B) P(\overline{A B} C)-P(A B C)(1-P(A) P(B)) \\
\leq P(A) P(B) P(\overline{A B C}) \\
\leq P(A) P(B) P(\overline{A B})=P(A) P(B)(1-P(A B)) \\
P(A \cup B)=P(A)+P(B)-P(A B) \leq 1, \\
P(A)+P(B)+1-P(A B) \leq 2 \\
P(A) P(B)(1-P(A B)) \\
\leq\left(\frac{P(A)+P(B)+1-P(A B)}{3}\right)^{3} \leq\left(\frac{2}{3}\right)^{3}=\frac{8}{27}, \\
\text { if } P(A)=P(B)=P(C)=\frac{2}{3}, \\
P(A B)=P(A C)=P(B C)=\frac{1}{3}, P(A B C)=0,
\end{gathered}
$$

the equality is true.

The proof is complete.

Proposition 6.

$$
-\left(\frac{n-1}{n}\right)^{n} \leq P\left(\mathrm{I}_{i=1}^{n} A_{i}\right)-\prod_{i=1}^{n} P\left(A_{i}\right)(n \geq 3)
$$

Proof.

$$
\begin{gathered}
\prod_{i=1}^{n} P\left(A_{i}\right)-P\left(\prod_{i=1}^{n} A_{i}\right) \\
=\left(\prod_{i=1}^{n-1} P\left(A_{i}\right)\right)\left(P\left(\prod_{i=1}^{n} A_{i}\right)+P\left(\prod_{i=1}^{n-1} A_{i} A_{n}\right)\right)-P\left(\prod_{i=1}^{n} A_{i}\right) \\
=P\left(\prod_{i=1}^{\overline{n-1} A_{i}} A_{n}\right)\left(\prod_{i=1}^{n-1} P\left(A_{i}\right)\right)-P\left(\prod_{i=1}^{n} A_{i}\right)\left(1-\prod_{i=1}^{n-1} P\left(A_{i}\right)\right) \\
\leq P\left(\prod_{i=1}^{n-1} A_{i} A_{n}\right)\left(\prod_{i=1}^{n-1} P\left(A_{i}\right)\right) \\
\leq P\left(\prod_{i=1}^{n-1} A_{i}\right)\left(\prod_{i=1}^{n-1} P\left(A_{i}\right)\right) \\
=\left(1-P\left(\prod_{i=1}^{n-1} A_{i}\right)\right)\left(\prod_{i=1}^{n-1} P\left(A_{i}\right)\right)
\end{gathered}
$$

We use inductive method to prove the conclusion as follows:

$$
\begin{gathered}
\left.\sum_{i=1}^{n-1} P\left(A_{i}\right)-P\left(I_{i=1}^{n-1} A_{i}\right)\right) \leq n-2 \\
\left.n=3, P\left(A_{1} \cup A_{2}\right)=\sum_{i=1}^{2} P\left(A_{i}\right)-P\left(\prod_{i=1}^{2} A_{i}\right)\right) \leq 1
\end{gathered}
$$

the inequality is true.

We assume that if $n=k$, then

$$
\left.\sum_{i=1}^{k-1} P\left(A_{i}\right)-P\left(I_{i=1}^{k-1} A_{i}\right)\right) \leq k-2,
$$

if $n=k+1$,

$$
\begin{aligned}
& P\left(\prod_{i=1}^{k} A_{i}\right)=P\left(\left(I_{i=1}^{k-1} A_{i}\right) I A_{k}\right) \\
= & P\left(\prod_{i=1}^{k-1} A_{i}\right)+P\left(A_{k}\right)-P\left(\left(\prod_{i=1}^{k-1} A_{i}\right) U A_{k}\right) \\
\geq & \sum_{i=1}^{k-1} P\left(A_{i}\right)-(k-2)+P\left(A_{k}\right)-P\left(\left(\prod_{i=1}^{k-1} A_{i}\right) U A_{k}\right) \\
\geq & \sum_{i=1}^{k} P\left(A_{i}\right)-(k-1) \\
& \left(P\left(A_{k}\right) \geq 0, \quad P\left(\left(\prod_{i=1}^{k-1} A_{i}\right) U A_{k}\right) \leq 1\right)
\end{aligned}
$$




$$
\sum_{i=1}^{k} P\left(A_{i}\right)-P\left(\left(_{i=1}^{k} A_{i}\right)\right) \leq k-1
$$

So,(7) is proved.

$$
\begin{gathered}
\left(1-P\left(\prod_{i=1}^{n-1} A_{i}\right)\right) \prod_{i=1}^{n-1} P\left(A_{i}\right) \\
\leq\left(\frac{1-P\left(\prod_{i=1}^{n-1} A_{i}\right)+\sum_{i=1}^{n-1} P\left(A_{i}\right)}{n}\right)^{n} \leq\left(\frac{n-1}{n}\right)^{n}, \\
-\left(\frac{n-1}{n}\right)^{n} \leq P\left(I_{i=1}^{n} A_{i}\right)-\prod_{i=1}^{n} P\left(A_{i}\right)
\end{gathered}
$$

if $P\left(A_{1}\right)=P\left(A_{2}\right)=\ldots=P\left(A_{n}\right)=\frac{n-1}{n}$,

$$
\left.\left.P\left(\mathrm{I}_{i=1}^{n-1} A_{i}\right)\right)=P\left(\prod_{\substack{i=1 \\ i \neq n-1}}^{n} A_{i}\right)\right)=\ldots=P\left(\left(_{i=2}^{n} A_{i}\right)\right)=\frac{1}{n} \text {, }
$$

$$
\prod_{i=1}^{n} A_{i}=\phi
$$

the equality is true.

We define $n$ events as follows:

$$
\begin{aligned}
& \left\{A_{i}, \mathrm{i}=1,2,3, \ldots \mathrm{n}\right\}: \\
& I=\{1,2,3, \ldots \mathrm{n}\} \\
& A_{1}=\{2,3, \ldots \mathrm{n}\}, A_{2}=\{1,3, \ldots \mathrm{n}\}, \ldots, A_{n}=\{1,2,3, \ldots, \mathrm{n}-1\} \\
& P\left(A_{1}\right)=P\left(A_{2}\right)=\ldots=P\left(A_{n}\right)=\frac{n-1}{n}, \\
& \left.\left.\left.P\left(I_{i=1}^{n-1} A_{i}\right)\right)=P\left(\prod_{\substack{i=1 \\
i \neq n-1}}^{n} A_{i}\right)\right)=\ldots=P\left(\prod_{i=2}^{n} A_{i}\right)\right)=\frac{1}{n}, I_{i=1}^{n} A_{i}=\phi, \\
& -\left(\frac{n-1}{n}\right)^{n} \leq P\left(I_{i=1}^{n} A_{i}\right)-\prod_{i=1}^{n} P\left(A_{i}\right) .
\end{aligned}
$$

The proof is complete.

\section{ACKNOWLEDGMENT}

This research is financially supported by three projects, the first fund project is Human and Social Science Funding Project granted by the Ministry of Education \#12YJA880124,"An Empirical Study of University Teachers under Engagement System --- from the perspective of international comparison", the second project is the project supported by the Fundamental Research Funds for the Central Universities (No.328201556),"Research on Graduate Mathematical Modeling and Innovation ability",the third project is the Central Universities in Beijing Joint Project supported by Beijing in 2015(research project:"talent cultivation joint project ---the famous teachers "),"Research on Academic Development Law of the famous teachers in universities in
Beijing--- based on the information collection and analysis of one hundred famous teachers career".

\section{REFERENCES}

[1] Xianping Li, Foundations of Probability (third edition). Beijing: higher education press, 2010,p.60.

[2] Xianping Li, Ziyi Chen, learning instruction on Foundations of Probability . Beijing: higher education press, 2011,p.36.

[3] Kai Lai Chung,:A Course in Probability theory (third edition) [M].Beijing:China Machine Press.2010.p.21. 